\title{
Forecasting Schizophrenia Incidence Frequencies Using Time Series Approach
}

\author{
Mohammad Ebrahim Ghaffari', Ali Ghaleiha ${ }^{2}$, Zahra Taslimi ${ }^{3,4}$, Fatemeh Sarvi ${ }^{1}$, Payam Amini ${ }^{5}$, Majid \\ Sadeghifar ${ }^{6}$, Saeid Yazdi-Ravandi ${ }^{2,3^{*}}$
}

${ }^{1}$ Department of Biostatistics \& Epidemiology, Hamadan University of Medical Sciences, Hamadan, Iran

${ }^{2}$ Behavioral Disorders and Substance Abuse Research Center, Hamadan University of Medical Sciences, Hamadan, Iran

${ }^{3}$ Young Researchers and Elite club, Rudehen Branch, Islamic Azad University, Rudehen, Iran

${ }^{4}$ Neurophysiology Research Center, Hamadan University of Medical Sciences, Hamadan, Iran

${ }^{5}$ Department of Epidemiology and Reproductive Health, Reproductive Epidemiology Research Center, Royan Institute for Reproductive Biomedicine, ACECR, Tehran, Iran

${ }^{6}$ Department of Statistics, Bu-Ali Sina University, Hamadan, Iran

\begin{abstract}
Background: Learning the prevalence of schizophrenia has main insinuations for both health service preparation and risk factor epidemiology. The aims of this research in order to systematically classify and collate studies explaining the prevalence of schizophrenia, to sum up the findings of these studies, also to survey selected factors that may influence occurrence approximates.

Methods: This historical cohort study was done on schizophrenia patients in Farshchian psychiatric hospital from April 2008 to April 2016. To analyze the data, the Holt-Winters Exponential Smoothing (HWES) method was applied. All the analyses were done by R.3.2.3. software using the packages "forecast" and "tseries." The statistical considerable level was simulated as 0.05 .

Results: Our investigation shows that a constant frequency of schizophrenia incidence happens every month from August 2008 to February 2015 while a considerable increase occurs in March 2015. The high frequency of schizophrenia incidence remains constant to the end of 2015 and a decrease is shown in 2016. Also, data demonstrate the development of schizophrenia in the next 24 months with $95 \% \mathrm{Cl}$.

Conclusion: Our study showed that a significant increase happens in the frequency of schizophrenia from 2016. Although the development is not constant and the same for all months, the amount of increase is considerably high compared to before 2016.

Keywords: Schizophrenia; Holt winter; Time series; Exponential smoothing; Modeling
\end{abstract}

\section{*Correspondence to} Saeid Yazdi-Ravandi, Ph.D. Student; Behavioral Disorders and Substance Abuse Research Center, Hamadan University of Medical Sciences, Hamadan, Iran.

Tel: +98(935)833 1910;

Email:

Saeid_Yazdiravandi@yahoo. com

Published online October 8, 2017

Citation: Ghaffari ME, Ghaleiha A, Taslimi Z, Sarvi F, Amini P, Sadeghifar M, et al. Forecasting schizophrenia incidence frequencies using time series approach. Int Clin Neurosci J. 2017;4(4):152-156. doi: 10.15171/icnj.2017.06.

\section{Introduction}

Schizophrenia is a disabling number of brain disorders characterized by symptoms such as hallucinations, delusions, disorganized interaction, poor planning, reduced incentive, and blunted influence. ${ }^{1}$ Schizophrenia is associated with deficits in various cognitive processes that result in disorders of complex thinking and ideation, resulting in difficulty in dealing with 'psychological and social challenges in daily life. ${ }^{2}$ Schizophrenia is a devastating psychiatric disorder that affects approximately $1 \%$ of the population. ${ }^{3}$ For several years, it was believed that the prevalence of schizophrenia varied little between sites. $^{4,5}$ It is, in fact, the case that the estimates of the prevalence of schizophrenia are seen as a multiplicity of variations. ${ }^{1,6-8}$ Major advancements have been made in our understanding of the epidemiology of schizophrenia. We now understand that the illness is somewhat more common and severe in young men, and that the occurrence may differ geographically and temporally. Risk factors have been elucidated; biological risks include a family history and ancestors of the complaint, advanced paternal age, obstetric difficulties, and abuse of drugs such as stimulants and cannabis. Furthermore, new research has also recognized social risk factors such as being born and brought up in a city, migration, and certain types of childhood difficulty such as physical misuse and bullying, as well as social separation and opposing events in adult lifetime. To conclude, the epidemiological evidence suggests that schizophrenia is a multifactorial disorder in which genes connect to the other person and with environmental factors to push individuals more than a threshold into expression of the disorder. ${ }^{9}$ Studies that estimate the occurrence of schizophrenia are required to be able to recognize gradients across time and/or place.

(C) 2017 The Author(s). This is an open-access article distributed under the terms of the Creative Commons Attribution-NonCommercial 4.0 International License (http://creativecommons.org/licenses/by-nc/4.0/) which permits copy and redistribute the material just in noncommercial usages, provided the original work is properly cited. 
These gradients allow us to generate prospect risk factors that may underlie difference in the disorder. However, studies that statement the prevalence of a disorder are also important. Estimating the amount of a population afflicted with schizophrenia is central to health service planning ${ }^{1}$ Assessing the stochastic process of a variable over time is of interest in lots of medical problems. ${ }^{10-12}$ Time series observations, as stochastic processes, are sequence of measurements of a special variable collected along with time. Lots of statistical methods have been introduced to analyze these types of data. Modeling and forecasting the development of a variable is a key characteristic of time series modeling approaches. Forecasting future conditions of variables cause a considerable reduction in costs and risks. ${ }^{13}$ The procedure of forecasting a future state of variable is carried out using present and previous conditions of the variable. ${ }^{14}$

Techniques to analyze these types of data can be determined based on some characteristics of time series such as stationary and/or seasonal observations. ${ }^{15}$ HoltWinters exponential smoothing (HWES) method is frequently used compared to other time series analysis methods thanks to its' efficacy. ${ }^{13,16}$

The purpose of this investigation is to assess monthly frequency of schizophrenia in Hamadan province from 2008 to 2016. Modeling and forecasting are carried out using HWES method.

\section{Materials and Methods \\ Data}

This historical cohort study was done on schizophrenia patients in Farshchian psychiatric hospital from April 2008 to April 2016. Patients according to psychiatrist diagnosed base on DSM-IV criteria were hospitalized. Also, data collection based on a checklist was developed by the investigators. To analyze the data, the HWES method was applied. All the analyses were done by R.3.2.3. software using the packages "forecast" and "tseries." The statistical considerable level was simulated as 0.05.

\section{Statistical Analysis}

\section{Holt-Winters Exponential Smoothing}

Time series data are stochastic processes in which the response variable, " $y$," is recorded on several occasions " $t . " 17$ A time series can be seasonal as well as stationary. Seasonal trend is described as the tendency of timeseries data to display behavior that reiterates itself every $\mathrm{L}$ periods. In a stationary time series, some properties such as mean and variance are constant over time. Several modeling approaches can be utilized according to stationarity and seasonality of the observation that is moving means method, linear regression with time, exponential smoothing. ${ }^{15}$ These models can be compared based on their goodness of fit and the accuracy of parameter estimations.

Exponential smoothing methods include single, double and triple (Holt-Winter) approaches and they can be used according to presence/absence of trend and seasonality. The HWES is applied when the series shows trend as well as seasonality. The HWES method models and forecasts the future condition of response variable by weighting the present and previous observation. This method allocates exponentially reducing weights as the prior surveillance get older. ${ }^{15}$ An additive or multiplicative seasonality in time series observations requires additive or multiplicative HWES approach, respectively.

An additive HWES takes the additive change in seasonal development of variable into account. An estimated amount is added to the beginning of the next period. The model includes " $y_{t+h}$ " as the frequency of Schizophrenia at " $t+h$ " (the time of processes), " $p$ " as period length, $\left(a t, b_{t}\right)$ as the permanent and trend of time series, as $S$ the additive seasonal factor and $\varepsilon_{t}$ as the random error component.

$$
y_{t+h}=a_{t}+h b_{t}+S_{t-p+1}+\epsilon_{t}
$$

Where

$$
\begin{aligned}
& a_{t}=\alpha\left(y_{t}-S_{t-p}\right)+(1-\alpha)\left(a_{t-1}+b_{t-1}\right) \\
& b_{t}=\beta\left(a_{t}-a_{t-1}\right)+(1-\beta) b_{t-1} \\
& S_{t}=\gamma\left(y_{t}-a_{t}\right)+(1-\gamma) S_{t-p}
\end{aligned}
$$

After the parameters in the model are estimated, one can forecast the response variable at any desired future time point by replacing the coefficients. All the analyses were done by statistical programming R.3.2.3. Software using "forecast" and "tseries" packages. The statistical significance level was simulated as 0.05 .

\section{Results}

This investigation assesses the frequency of schizophrenia in Hamadan province, west of Iran, from August 2008 to February 2016. According to Figure 1, a constant frequency of schizophrenia incidence happens every month from August 2008 to February 2015 while a considerable increase occurs in March 2015. The high frequency of schizophrenia incidence remains constant to the end of 2015 and a decrease is shown in 2016.

The additive HWES method was performed to model the frequency of schizophrenia incidence. The dashed line in Figure 1 shows the estimated frequencies by the HWES. The model is as follows.

$$
y_{t+h}=a_{t}+h b_{t}+S_{t-p+1}
$$

Where

$$
\begin{aligned}
& a_{t}=0.43\left(y_{t}-S_{t-p}\right)+0.57\left(a_{t-1}+b_{t-1}\right) \\
& b_{t}=0.03\left(a_{t}-a_{t-1}\right)+0.97 b_{t-1} \\
& S_{t}=0.35\left(y_{t}-a_{t}\right)+0.65 S_{t-p}
\end{aligned}
$$

The level, trend and seasonal components are estimated 


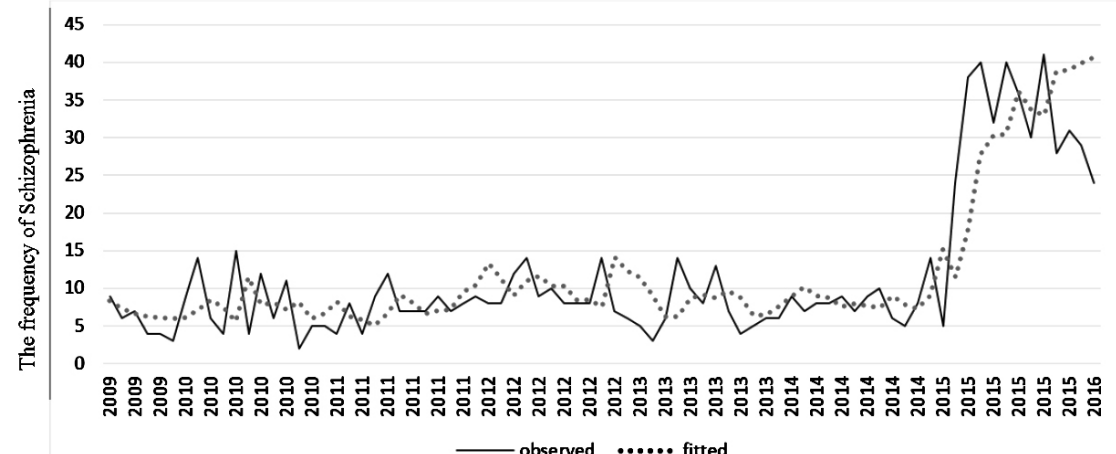

Figure 1. The Development of Schizophrenia Frequency (Dashed line =estimated frequencies resulted by the HWES, line $=$ Observed frequency).

and shown in Table 1 while the period is 12 months $(p=12)$. The estimated HWES model was applied to forecast the Schizophrenia incidence frequencies for the next 24 months after February 2016. The frequencies as well as a 95\% CI are exposed in Table 2. In addition, Figure 2 demonstrates the development of schizophrenia in the next 24 months with 95\% CI. According to Figure 2, a constant behavior of schizophrenia incidence frequencies occurs from March 2016 to February 2017 with an average of 41 frequencies each month. An increase happens in March 2017 and ends in October 2017 with an average of 54 schizophrenic patients each month. The schizophrenia incidence frequencies decreased after November 2017.

\section{Discussion}

The history of the epidemiological study of schizophrenia is as old as the diagnostic concept itself. The worldwide incidence of schizophrenia reported according to DSM standard, and modified for age, mean occurrence is 0.11 (range 0.07-0.17) per 1000 inhabitants per year. ${ }^{16}$ Estimations of the lifetime frequency of schizophrenia range from $0.4 \%$ to $1.4 \%$. The first age of onset and the persistent illness course may demonstrate these

Table 1. The Estimation of Level, Trend and Seasonal Components of Time Series HWES Model

\begin{tabular}{lc}
\hline Coefficients & Estimates \\
\hline a & 39.129 \\
b & 0.738 \\
s1 & 1.051 \\
s2 & 1.093 \\
s3 & 1.258 \\
s4 & 1.061 \\
s5 & 0.998 \\
s6 & 1.169 \\
s7 & 0.947 \\
s8 & 0.953 \\
s9 & 0.726 \\
s10 & 0.775 \\
s11 & 0.712 \\
\hline
\end{tabular}

respectively high characters. Patients with schizophrenia may also have limited usage of and lower quality of healthcare services contrasted with the public population. ${ }^{18}$ It really is clear that our knowledge of rate of recurrence of the disorder has improved substantially during the past few decades with evidence recommending that some previously organized views about its occurrence were not

Table 2. A 24 Months Forecasting of Schizophrenia Using the HWES Model

\begin{tabular}{|c|c|c|}
\hline Date & Point Forecast & $95 \% \mathrm{CI}$ \\
\hline 16-Mar & 41.938 & $35.545-48.330$ \\
\hline 16-Apr & 44.384 & $36.467-52.301$ \\
\hline 16-May & 52.014 & 42.093-61.935 \\
\hline 16-June & 44.659 & $34.717-54.601$ \\
\hline 16-July & 42.742 & $32.099-53.385$ \\
\hline 16-Aug & 50.929 & $37.733-64.125$ \\
\hline 16-Sep & 41.996 & $29.865-54.127$ \\
\hline 16-Oct & 42.937 & $29.697-56.178$ \\
\hline 16-Nov & 33.239 & $21.610-44.868$ \\
\hline 16-Dec & 36.075 & $22.737-49.414$ \\
\hline 17-Jan & 33.661 & $20.233-47.089$ \\
\hline 17-Feb & 31.150 & $18.080-44.219$ \\
\hline 17-Mar & 51.264 & $28.953-73.576$ \\
\hline 17-Apr & 54.075 & $29.992-78.158$ \\
\hline 17-May & 63.167 & $34.705-91.629$ \\
\hline 17-June & 54.067 & $28.774-79.360$ \\
\hline 17-July & 51.591 & $26.713-76.468$ \\
\hline 17-Aug & 61.295 & $31.454-91.135$ \\
\hline 17-Sep & 50.401 & 24.894-75.908 \\
\hline 17-Oct & 51.389 & $24.791-77.988$ \\
\hline 17-Nov & 39.676 & $17.997-61.356$ \\
\hline 17-Dec & 42.951 & $19.036-66.865$ \\
\hline 18-Jan & 39.976 & $16.928-63.024$ \\
\hline 18-Feb & 36.904 & $15.172-58.636$ \\
\hline
\end{tabular}




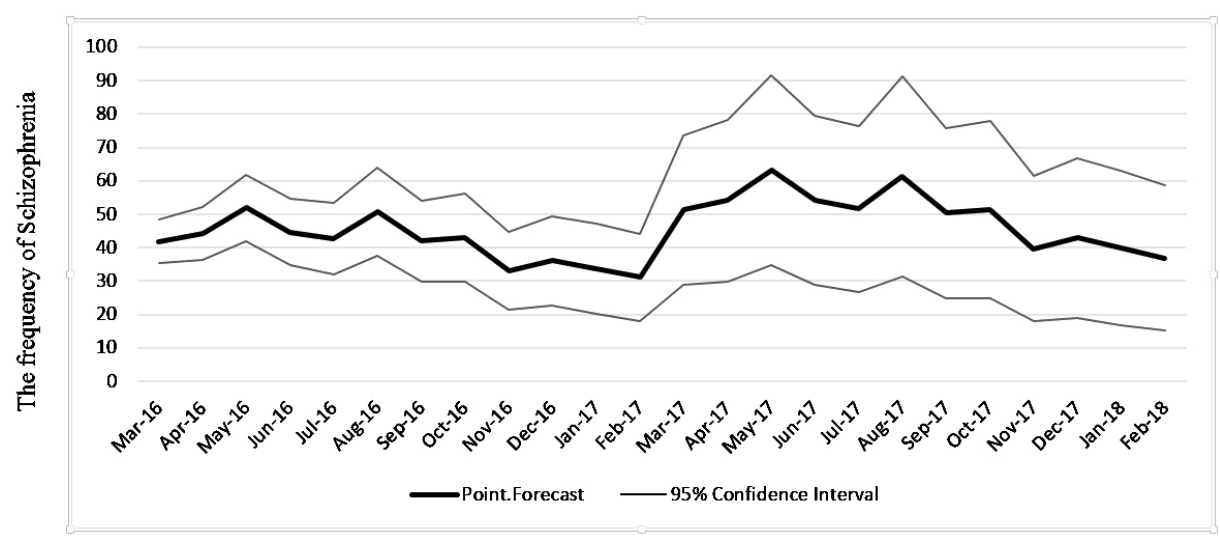

Figure 2. Forecasting 24 Next Months of Schizophrenia Frequency From March 2016 to February 2018.

exactly appropriate. Still, there remain important gaps in the literary works that can only be filled with more population-based studies using widely conventional and validated ascertainment tools. ${ }^{18}$ Nevertheless, it should be mentioned that the boundaries chosen for epidemiological studies (e.g., health districts, towns, states, or nations) might not exactly be optimal for the detection of differences of the disorder within or between various populations. Studies that estimate occurrence of schizophrenia are essential in order to identify gradients across time and/or place. These gradients allow all of us to create prospect risk factors that may underlie different versions in the disorder. On the other hand, studies that report the prevalence of the disorder are also important. ${ }^{1}$ Generally, epidemiologic research has built a powerful knowledge base over the past quarter hundred years, and this knowledge foundation will continue to make contributions to public well-being efforts at prevention of schizophrenia in the coming decades. ${ }^{19}$ We used HWES method to model and forecast the schizophrenia incidence frequencies using the data from 91 months from 2008 to 2016. Lots of studies have used the HWES method with both additive and multiplicative approaches to analyze time series data in medical areas. ${ }^{20-23}$ Limitations of this study are that the study was done according to the patient's records in hospital and we did not have access to information before 2008 .

Our study showed that a significant increase happens in the frequency of schizophrenia from 2016. Although the development is not constant and the same for all months, the amount of increase is considerably high compared to before 2016. The HWES method can be used as an appropriate statistical tool to analyze and forecast such data.

\section{Conflict of Interest Disclosures}

The authors declare that they have no conflict of interests.

\section{Ethical Statement}

The research was approved by Hamadan University of Medical Sciences Local Ethical Committee (No. 94047091977).

\section{Acknowledgment}

We all thank the Vice-chancellor of Health Services of Hamadan University of Medical Sciences for providing us the dataset. Furthermore, we would like to thank the Vice-chancellor of Research and Technology of Hamadan University of Medical Sciences for financial support of this investigation (grant No. 940470919977)

\section{References}

1. Saha S, Chant D, Welham J, McGrath J. A systematic review of the prevalence of schizophrenia. PLoS Med. 2005;2(5):e141. doi: 10.1371/journal.pmed.0020141.

2. Deshpande SN, Bhatia T, Mohandas E, Nimgaonkar VL. Cognitive remediation in schizophrenia-The view from India. Asian J Psychiatr. 2016;22:124-8. doi: 10.1016/j. ajp.2016.06.011.

3. Donegan JJ, Lodge DJ. Cell-based therapies for the treatment of schizophrenia. Brain Res. 2017;1655:262-9. doi: 10.1016/j. brainres.2016.08.010.

4. McGrath JJ, Susser ES. New directions in the epidemiology of schizophrenia. Med J Aust. 2009;190(4 Suppl):S7-9.

5. Jablensky A. Epidemiology of schizophrenia: the global burden of disease and disability. Eur Arch Psychiatry Clin Neurosci. 2000;250(6):274-85.

6. Saha S, Chant D, McGrath J. Meta-analyses of the incidence and prevalence of schizophrenia: conceptual and methodological issues. Int J Methods Psychiatr Res. 2008;17(1):55-61. doi: 10.1002/mpr.240.

7. Kirkbride JB, Fearon P, Morgan C, Dazzan P, Morgan K, Tarrant $\mathrm{J}$, et al. Heterogeneity in incidence rates of schizophrenia and other psychotic syndromes: findings from the 3-center AeSOP study. Arch Gen Psychiatry. 2006;63(3):250-8. doi: 10.1001/ archpsyc.63.3.250.

8. Esan O. Prevalence of schizophrenia: recent developments: letter to the editor. African Journal of Psychiatry. 2013;16(2):93. doi: 10.4314/ajpsy.v16i2.

9. van Os J, Marcelis M. The ecogenetics of schizophrenia: a review. Schizophr Res. 1998;32(2):127-35.

10. Caballero Barajas K, Akella R. Dynamically Modeling Patient's Health State from Electronic Medical Records: A Time Series Approach. In: Proceedings of the 21th ACM SIGKDD International Conference on Knowledge Discovery and Data Mining 2015; 69-78.

11. Weinberger DM, Klugman KP, Steiner CA, Simonsen L, Viboud C. Association between respiratory syncytial virus activity and pneumococcal disease in infants: a time series analysis of US 
hospitalization data. PLoS Med. 2015;12(1):e1001776. doi: 10.1371/journal.pmed.1001776.

12. Dayer MJ, Jones S, Prendergast B, Baddour LM, Lockhart PB, Thornhill MH. Incidence of infective endocarditis in England, 2000-13: a secular trend, interrupted time-series analysis. Lancet. 2015;385(9974):1219-28. doi: 10.1016/s01406736(14)62007-9.

13. Kalekar PS. Time series forecasting using holt-winters exponential smoothing. Kanwal Rekhi School of Information Technology. 2004;4329008:1-3.

14. Box GE, Jenkins GM, Reinsel GC, Ljung GM. Time Series Analysis: Forecasting and Control. New Jersy: John Wiley \& Sons; 2015.

15. Benedict LH, Gould RD. Towards better uncertainty estimates for turbulence statistics. Exp Fluids. 1996;22(2):129-36. doi: 10.1007/s003480050030.

16. Pan R. Holt-Winters Exponential Smoothing. In: Wiley Encyclopedia of Operations Research and Management Science. John Wiley \& Sons, Inc; 2010.

17. Pan R, Cochran JJ, Cox LA, Keskinocak P, Kharoufeh JP, Smith JC. Holt-Winters Exponential Smoothing. In: Wiley Encyclopedia Operations Research Management Science. John Wiley Sons, Inc; 2010.

18. Hamilton JD. Time Series Analysis. Princeton: Princeton University press; 1994.

19. Szkultecka-Debek M, Walczak J, Augustynska J, Miernik
K, Stelmachowski J, Pieniazek I, et al. Epidemiology and treatment guidelines of negative symptoms in schizophrenia in central and eastern Europe: a literature review. Clin Pract Epidemiol Ment Health. 2015;11:158-65. doi: 10.2174/1745017901511010158.

20. Messias EL, Chen CY, Eaton WW. Epidemiology of schizophrenia: review of findings and myths. Psychiatr Clin North Am. 2007;30(3):323-38. doi: 10.1016/j. psc.2007.04.007.

21. Murray N. Forecasting Monthly Incidence Rates for Shigellosis in Tennessee [Thesis]. Knoxville, USA: University of Tennessee; 2014.

22. Moore PJ, Little MA, McSharry PE, Geddes JR, Goodwin GM. Forecasting depression in bipolar disorder. IEEE Trans Biomed Eng. 2012;59(10):2801-7. doi: 10.1109/ TBME.2012.2210715

23. Dorea FC, McEwen BJ, McNab WB, Revie CW, Sanchez J. Syndromic surveillance using veterinary laboratory data: data pre-processing and algorithm performance evaluation. J R Soc Interface. 2013;10(83):20130114. doi: 10.1098/ rsif.2013.0114.

24. Zhang L, Zheng Y, Wang K, Zhang X, Zheng Y. An optimized Nash nonlinear grey Bernoulli model based on particle swarm optimization and its application in prediction for the incidence of Hepatitis B in Xinjiang, China. Comput Biol Med. 2014;49:67-73. doi: 10.1016/j.compbiomed.2014.02.008. 